\title{
The Application of Combination Method of CBL and PBL in Applied Disciplines —A Case Study of "Food Sensory Analysis"
}

\author{
Hongwei Wang \\ College of Food Science, Southwest University, Chongqing, China \\ wanghwljl@qq.com
}

Keywords: CBL; PBL; Applied Disciplines.

\begin{abstract}
In order to avoid the shortcomings of the traditional teaching method in applied disciplines, based on the curriculum teaching reform of food sensory analysis, this paper discussed the application of case-based learning (CBL) and problem-based learning (PBL) in applied disciplines.
\end{abstract}

\section{Introduction}

The goal of professional teaching of applied disciplines is to cultivate high-level practical talents with innovative practice ability, while the core of applied disciplines teaching reform is to attach importance to and strengthen practice teaching. In the process of professional teaching of applied disciplines, special attention need to pay to guide students to think things out for themselves, practice autonomously and focus on cultivating students' innovative spirit and professional practical ability. Food sensory analysis is also known as food sensory evaluation, Sensory evaluation has been defined as a scientific method used to evoke, measure, analyze, and interpret those responses to products as perceived through the senses of sight, smell, touch, taste, and hearing[1]. Food sensory analysis is a new developed discipline based on the physical and chemical analysis of food as well as the combination of psychology, physiology and statistics knowledge. The field of sensory evaluation grew rapidly, along with the expansion of the processed food and consumer products industries. Sensory evaluation comprises a set of techniques for accurate measurement of human responses to foods and minimizes the potentially biasing effects of brand identity and other information influences on consumer perception. As such, it attempts to isolate the sensory properties of foods themselves and provides important and useful information to product developers, food scientists, and managers about the sensory characteristics of their products [2]. As a typical applied discipline, food sensory analysis is becoming an important technical support for modern food science and technology as well as the development of the food industry.

Although the course "food sensory analysis" was widespreadly set up to the udergraduate in food science and engineering, food quality and safety in China, this curriculum teaching is still at the exploratory stage. Some colleges even only set up the theoretical courses, while ignore the practice. Food sensory analysis based on human senses, so that the subjective and objective factors, such as the environmental conditions, physical condition and mood of the panelists, would affect the results. Therefore, it is difficult for students to master the course just through traditional lecture-based learning method. How to improve the teaching effect, to meet the needs of practice and research, is a problem that need to explore for quite a long time.

With the popularity of "student-centered, teacher-led" new learning model, the case based learning (CBL), problem based learning (PBL), and their combination has been widely used in recent years. However, new learning model mainly applied in the field of medical education [3-9]. The characteristic of CBL teaching method is that teachers and students should share the responsibility and guide students to explore problems, identify problems and solve problems through the preparation. Its core takes case as guidance, problem based, student as the main body, teacher led. It is characterized by helping students to master relevant knowledge, mobilize students' enthusiasm, and 
cultivate students' ability of self-learning and innovation through real cases. By presenting cases, the teacher guides students to solve the practical problems by using the studied theoretical knowledge. PBL teaching method is teacher led, problem centered and it takes students as the main body of teaching activities. By designing real tasks, students accessing information, analyzing and discussing over the question and solve the problem through their independent inquiry and cooperation. In this process, teachers offering their guide and key questions answer to enable students to achieve their goals. CBL and PBL have their own advantages and disadvantages, the combination of the two can complement each other, to achieve better teaching effect. "Food sensory analysis" course has theoretical, practical and technical characteristics, which is quite suitable for the adopting the combination of CBL and PBL in teaching.

\section{Aims and Strategy}

\section{Adjust the Teaching Content, Adapt to the Needs of Discipline Development}

At present, "food sensory analysis" course mostly will last about 30-40 classes in China, which include both class teaching and practice. The main content of this course includes physiological and psychological foundations of sensory function, principles of good practice, discrimination testing, descriptive analysis, affective test. It is difficult for students to master all knowledge. Therefore, in order to improve syllabus, promote students' ability and quality, it is imperative to adjust and reform the teaching content of curriculum.

According to the graduate courses, as well as the "food sensory analysis" course, the main content to undergraduate should be focused on basic application and practice. As for sensory analysis section based on examples, the operation method, the scope of application and how to use software to analyze the data are mainly explained. The strong theoretical design and statistical processing principle would be postponed to the postgraduate stage. It reduced the students' fears in learning data analysis, strengthened the practical application and improved students' confidence and enthusiasm. Through the adjustment of the content, there will be more class for the experiment to improve the students' practical ability.

As for teaching content, we can also connect "food sensory analysis" course with other related courses as "fruit and vegetable processing technology", "grain and oil processing technology", "animal product processing technology", "brewing technology", "soft drink technology" and so on. Applying the principle and method of food sensory analysis, to evaluate the quality of related course experiment produced food and forecast its shelf life which will enhance students' mastery of the related curriculum knowledge through keenly experience these differences in food sensory quality. For example, when the students descriptive the attributes of different yoghurts which are cultived on different fermentation strains, fermentation temperature, fermentation time and food thickener, it will deepen the students' mastery of yogurt fermentation process.

Food sensory analysis is not only a science subject, but also a practical technology. When students understanding and mastering the theoretical system, they are more concerned about the application of food sensory analysis in their daily life and practical work at the same time. According to the above characteristics of this course, based on syllabus, the teaching content has increased the sensory analysis application of all kinds of food and tea, expanded the teaching content and strengthened the practical application of the course. For example, sensory quality indicators and identification methods of several common fruits and vegetables, flavor characteristics and evaluation of different wines, flavor characteristics and evaluation of tea etc. In addition, some dietary culture, wine culture and tea culture related to sensory evaluation were lectured. For example, sensory analysis is useful to explain the characterics of green tea, black tea, oolong tea, dark tea, yellow tea, white tea these six different processing tea. These contents are closely conected with common life, and help students find out the curriculum is useful. 


\section{Innovate Teaching Methods to Improve Teaching Effect}

The experimental part in "food sensory analysis" course accounted an important proportion. Considering to the number of laboratory sensory evaluation compartments, a small class teaching of 20 people or so is generally adopted. The course of "food sensory analysis" includes two parts: theoretical explanation and practical operation. Theoretical explanation section not only has the theory based knowledge: physiological and psychological foundations of sensory function, environmental conditions for sensory analysis, panel building and samples preparation, but also has the specific methods based applications, such as discrimination testing, descriptive analysis, affective test. The former is suitable for the existing traditional lecture-based learning (LBL) to teach in class, the latter is suitable for adapting CBL method and combined with experimental operation to explain in laboratory. Practical operation mainly using PBL method.

When using the LBL method to teach basic knowledge, pay attention to increase the picture, video content, improve the visibility of the course, so that the content would be more intuitive, easier for students to understand, also gives people aesthetic enjoyments. For example, if there is not a standard sensory analysis laboratory, therefore, combined with real pictures or video of foreign advanced sensory analysis laboratory, tell about the division of each region, equipment configuration and environment requirements of sensory analysis laboratory. What's more, combined with specific examples, tell about the influence of various designs on sensory analysis so that students could have intuitive sensations. When lecturing the selection and training of assessors in descriptive analysis, taking the scene photos of the assessor training as an example, let the students earnestly feel the atmosphere of training.

When lecturing the sensory analysis methods such as discrimination testing, descriptive analysis, affective test, use CBL method to test the students as the evaluation personnel, then the students evaluation results as a case study to explain the data analysis and results discussion. Diving students into two groups and prepare samples for each other so that students can be keenly aware of different sensory analysis methods, also grasp the requirements of each method for sample preparation and more comprehensively grasp the sensory analysis methods. Due to the different coding of the two groups, at the time of evaluation, students are unable to the sample information of the other group, which avoid the interference caused by self-sample preparation. At the time of the discussion, analysing these data will help students to grasp these methods. It would be easier for students to better understand and master various sensory analysis methods that explaining how various methods operating and teaching principles and applications in examples. In addition, through their own operations and discussions, students acquired the experimental skills and data processing methods, with lively and vivid teaching process. Compared with the pure teaching, the teaching effect has been greatly improved.

The PBL method is mainly used in the experiment teaching, which is to guide the students to discuss and design the experimental verification independently around the scientific research or life problems. The previous experimental teaching mainly was teacher prepare the experiment contents for students according to the experimental requirements, students just comment according to the teacher's instructions in class. After the experiment, the students have a lack of understanding of food sensory analysis, many students still think that food sensory analysis is very simple, only need to taste the food, smell the aroma then fill in a few simple forms, which is far less than the purpose of the experimental class. Therefore, it is necessary for the students to participate in the whole preparation, the implementation process and the final statistical report. Students are required to set up a group of 5-6 students that would do one study such as "consumers' preference test of different concentrations of orange juice", "discrimination test of commercially available yogurt texture", "discrimination test of different brands tea drinks", "study on the effect of salt on the watermelon juice sweetness". Students had to complete a series of experimental processes by themselvies, such as experimental design, evaluation procedure, draw a conclusion through data anlysis. Finally, each group was required to make summary and analysis in class. If the experiment obtains the anticipated result, let the student to analyze the successful key step and the experience. If not, let the student analyze the 
reasons for the failure of experiment and propose the improvement opinions. In this way, not only the students attach great importance to the experimental class, but also cultivate the students' ability to analyze and solve problems and lays the foundation for future research papers. For example, when students studying the effect of salt on watermelon juice sweetness, through pre-experiment, it was found that the sweetness of watermelon juice was significantly different after added salt. Then further study the effect of salt concentration on the sweetness of watermelon juice, the results showed that when the salt additive amount of $100 \mathrm{~g}$ watermelon juice was higher than $0.037 \mathrm{~g}$, the sweetness of watermelon juice was obviously enhanced. When testing "commercially available differential test of different brands tea drinks", students had A- not- A test experiment in four classes respectively; it was found that the experimental results of each class were not significantly different. While analyzing the experimental results of all assessors, the results were significantly different. Students could combine theoretical knowledge independently figure out the number of panel on experimental results and statistical results. In each experiment, the students showed great enthusiasm and enthusiasm with consistent reflection of great experimental harvests.

In the course of consulting literature, designing and implementing data and experimental scheme, students not only comprehensively used the learned knowledge but also understand and master the relevant knowledge to design a feasible experimental scheme. Setting up comprehensive experiments can cultivate students' creative thinking ability and innovation consciousness.

\section{Guide Students to Participate in Scientific Research, Make Practical Use of Knowledge}

In order to strengthen the cultivation of students' ability and improve the students' ability to apply professional knowledge, in recent years, the author has recruited the undergraduate who are interested in sensory analysis to participate in the scientific research. The author establish a student panel to describe the attributes of steamed cake, yogurt and other foods. Through the study of these projects, students not only applied their theoretical knowledge to the research, but also expanded their knowledge, which is helpful to improve their experimental ability, cultivate their innovation ability and greatly improve their comprehensive quality.

In the past few years, the author also encouraged students to carry out their graduate projects or innovation projects and explain the projects in "food sensory analysis" course. This not only urges students to learn, increase the practicality, but also produce more contents and forms. Moreover, in the process of production practice and graduation practice, the author also encourages students to use the food sensory analysis knowledge to solve practical problems in production.

\section{Conclusion}

"Food sensory analysis" course adopts the mode of combining theory with practice closely. In addition to the traditional LBL method, combining CBL method with PBL method was used to stimulate the students' desire to learn which obtained good teaching effect. Through the reform of curriculum teaching content, strengthen the training of experiment teaching, lay stress on applications of sensory analysis in practice, which could effectively mobilize students' enthusiasm, improve students' basic skills and cultivate students' innovation ability. Combined with professional characteristics, introducing students into the research work and developing students' ability to apply knowledge and scientific research ability. The course content could be extended from service to the production practice and the graduation practice, further improve students' ability to analyze problems and solve practical problems and it plays a positive role in improving the overall quality and comprehensive ability of students.

\section{Acknowledgements}

This research was supported by education and teaching reform research project (2015JY056) and teaching team construction of basic courses of food science in Southwest University. 


\section{References}

[1] H. Stone, J.L. Sidel, Sensory evaluation practices, third edition. Academic, San Deigo, 2004.pp. $1-19$.

[2] H.T. Lawless, H. Heymann, Sensory evaluation of food: principles and practices, second edition. Springer, New York, 2010.pp.1-18.

[3] M. Andrews, P.R. Jones, Problem-based learning in an undergraduate nursing programme: A case study, Journal of Advanced Nursing. 23(1996) 357-365.

[4] K. Prince, H. van Mameren, N. Hylkema, Does problem-based learning lead to deficiencies in basic science knowledge? An empirical case on anatomy, Medical Education. 37(2003):15-21.

[5] J.L. Kolodner, P.J. Camp, D. Crismond, Problem-based learning meets case-based reasoning in the middle-school science classroom: Putting Learning by Design (TM) into practice, Journal of the Learning Sciences. 12(2003):495-547.

[6] T. Balslev, W.S. de Grave, A. Muijtjens, A. Scherpbier, Comparison of text and video cases in a postgraduate problem-based learning format, Medical Education. 39(2005):1086-1092.

[7] B. Williams, Case based learning - a review of the literature: is there scope for this educational paradigm in prehospital education? Emergency Medicine Journal. 22(2005):577-581.

[8] M. Srinivasan, M. Wilkes, F. Stevenson, T. Nguyen, S. Slavin, Comparing problem-based learning with case-based learning: Effects of a major curricular shift at two institutions, Academic Medicine. 82(2007):74-82.

[9] F. Mosannenzadeh, A. Bisello, C. Diamantini, G. Stellin, D. Vettorato, A case-based learning methodology to predict barriers to implementation of smart and sustainable urban energy projects, Cities. 60(2017):28-36. 